\title{
Correction to: How Quantum is Quantum Counterfactual Communication?
}

\author{
Jonte R. Hance ${ }^{1}$ (D) . James Ladyman ${ }^{2}$. John Rarity ${ }^{1}$
}

Published online: 24 March 2021

(c) Springer Science+Business Media, LLC, part of Springer Nature 2021

\section{Correction to: Foundations of Physics (2021) 51:12 https://doi.org/10.1007/s10701-021-00412-5}

The original version of this article contains mistake in the figures placement. The image file for Fig. 3 is being used for Fig. 4, the image file for Fig. 4 is being used for Fig. 5 and the image file for Fig. 5 is being used for Fig. 3. The correct Figs. 3, 4, 5 with figure caption is given below. The original article has been corrected.

The original article can be found online at https://doi.org/10.1007/s10701-021-00412-5.

Jonte R. Hance

jonte.hance@bristol.ac.uk

1 Quantum Engineering Technology Laboratories, Department of Electrical and Electronic Engineering, University of Bristol, Woodland Road, Bristol BS8 1US, UK

2 Department of Philosophy, University of Bristol, Cotham House, Bristol BS6 6JL, UK 


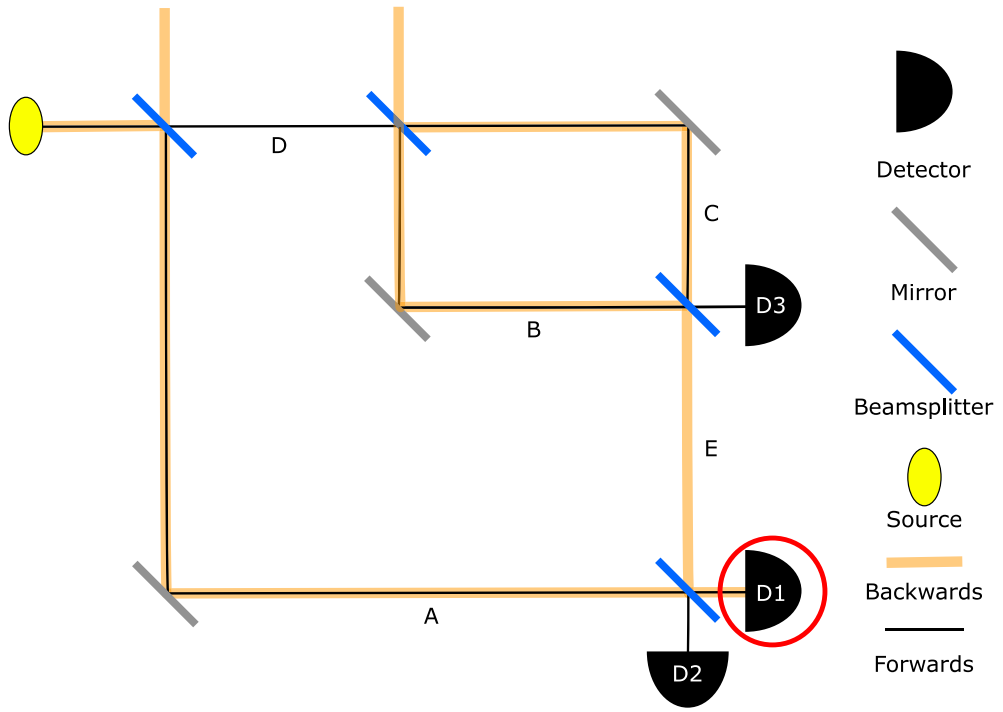

Fig. 3 The Two-State Vector formalism applied to a nested interferometer. Forwards-travelling paths are marked by thin black lines, and backwards-travelling paths by thick orange lines. Though no forward or backwards travelling path goes from the source, to Bob (along path C) and into D2, they do overlap over $\mathrm{C}$, meaning there is a weak trace at Bob. This illustrates the peculiar property of the TSVF where particles can jump between regions (e.g. between the inner interferometer and the outer arm) [51]

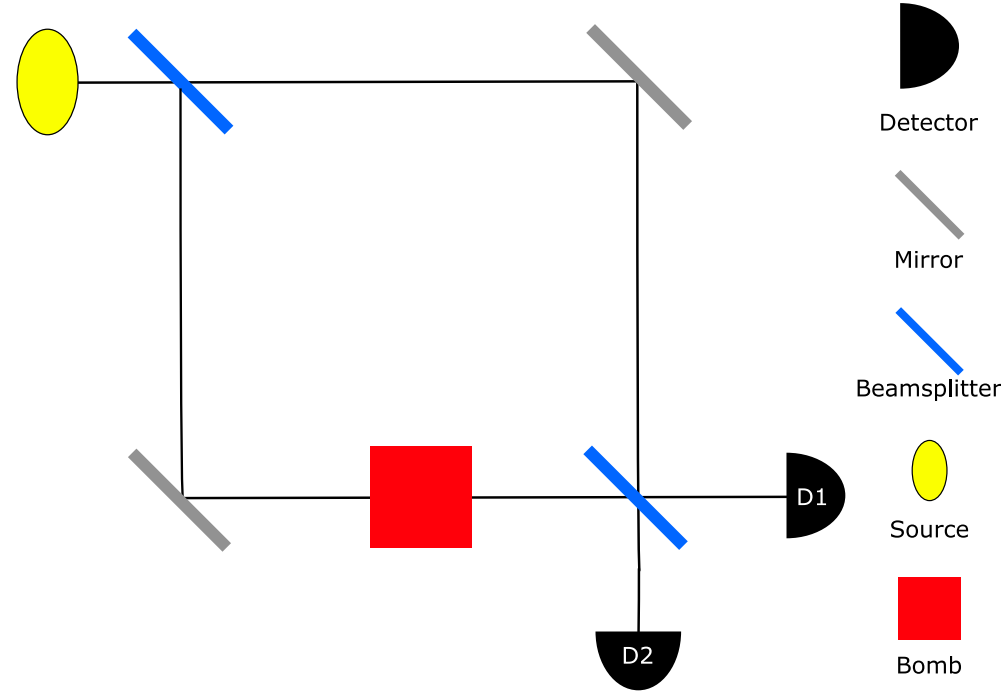

Fig. 4 The Elitzur-Vaidman Bomb Tester. A photon is emitted from the source (top-left), enters the balanced Mach-Zehnder interferometer, and is spread across both paths equally. If the bomb is faulty, the photon recombines at the second beam-splitter, and always enters $D_{1}$. If the bomb works, and is activated, it destroys the set-up. If the bomb would work, but the photon went down the bomb-free path, the photon has a 50:50 chance of going to either detector 


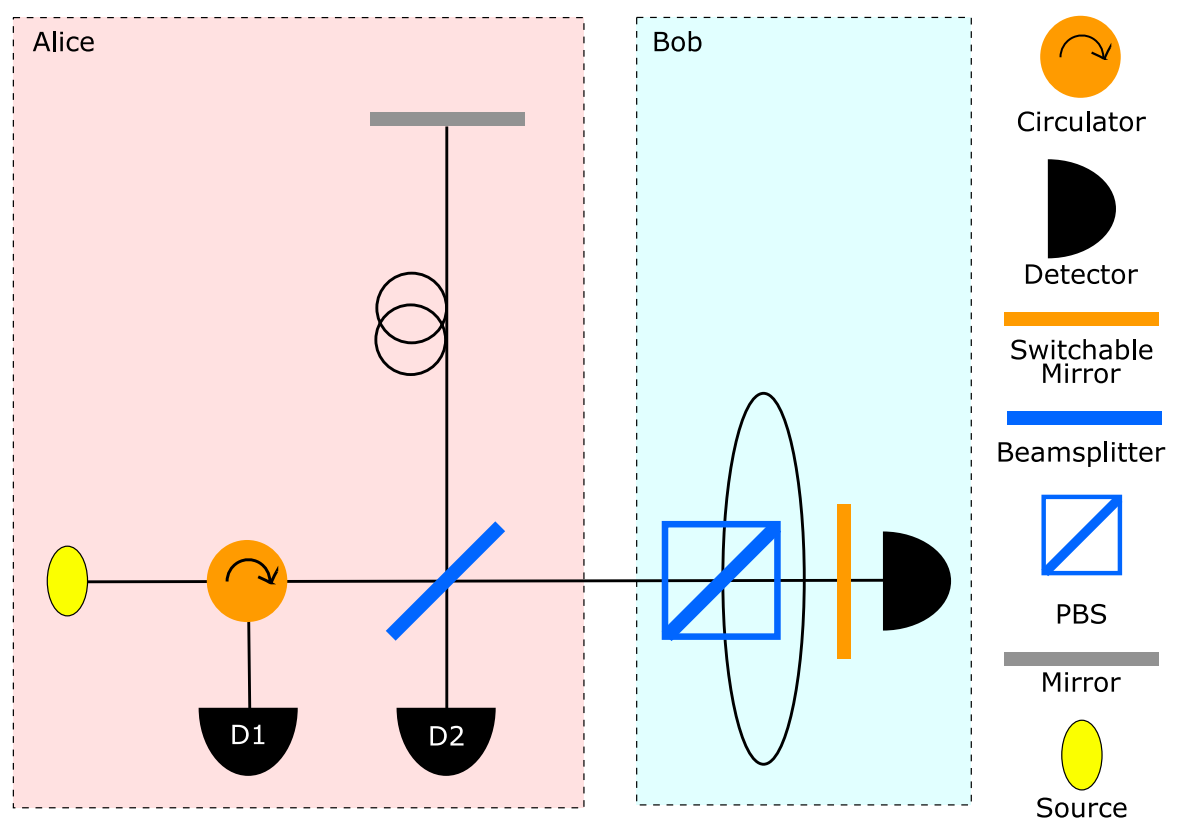

Fig. 5 Noh's counterfactual cryptography protocol-Alice randomly polarises a photon, which passes through a beam-splitter, with one of the outputs going to Bob. Bob uses a PBS and delay to time-separate possible polarisations arriving it him, and picks one to reflect and one to absorb. If the photon is Bob's reflect-polarisation, it reflects back, and interferes into Alice's $D_{2}$. However, if the photon is his absorbpolarisation, it is sent into his detector. If this clicks, the protocol is aborted; if not, the photon goes into $D_{1}[31]$

Publisher's Note Springer Nature remains neutral with regard to jurisdictional claims in published maps and institutional affiliations. 